\title{
A MAC Protocol to Reduce Sleep Latency and Collisions in Wireless Sensor Network
}

\author{
Jinsuk Pak, Jeongho Son, and Kijun Han* \\ Department of Computer Engineering, Kyungpook National University, \\ 1370, Sankyuk-dong, Puk-gu, Daegu, 702-701, Korea \\ \{jspak, jhson\}@netopia.knu.ac.kr, kjhan@knu.ac.kr
}

\begin{abstract}
This paper presents a MAC protocol which uses separate wakeup slots for each sensor node in sensor networks. Most MAC protocols proposed for sensor network are inefficient under heavy traffic loads, in particular in high density network topology because of frequent collisions and long sleep latency. In this paper, we suggest a MAC protocol in which each node has a different wakeup schedule in the same duty cycle, and it joins the competition only for its own short wakeup slot when the receiver is ready to receive its data. Simulation results indicate that our scheme can reduce energy consumption and minimize idle listening which increases the power efficiency.
\end{abstract}

\section{Introduction}

In Wireless Sensor Networks (WSN), energy efficiency is one the most critical issues in order to prolong network lifetime since it is often not feasible to replace or recharge batteries for sensor nodes. Media Access Control (MAC) protocols must minimize the radio energy costs in sensor nodes. Latency and throughput are also important design features for MAC protocols in WSN [3].

The SMAC proposed in [2], which is a modified version of the IEEE 802.11 Distributed Coordinator Function (DCF), provides a tunable periodic active/sleep cycle for sensor nodes. It puts nodes to sleep at certain times to conserve energy [4]. However, periodic sleep may result in a long sleep latency since the sending node has to wait until the receiving node wakes up in its listen period. Timeout MAC (TMAC) alleviated the problems of SMAC by using an adaptive duty cycle. In TMAC, if a node does not observe any activity in the neighborhood for some time, it goes to sleep early. TMAC saves more energy under variable traffic loads, but it still has problems of long sleep latency and low throughput.

We propose a new MAC protocol to solve the sleep delay and collision problems by allocating different listen period to each node in the same duty cycle with a legacy sensor MAC protocol. In our MAC protocol, each sensor node joins the competition only for its own short wakeup slot when the receiver is ready to receive its data. Our MAC protocol can reduce the possibility of collision and decrease sleep delay due to contention. Also, it has a shorter listen period than SMAC, which contributes to reducing energy waste and thus to improving the power efficiency.

\footnotetext{
* Corresponding author.
} 


\section{Our MAC Protocol}

In this paper, we propose a MAC protocol to reduce collisions and decrease latency caused by periodic sleeping. As shown in Fig. 1, in our MAC protocol, a superframe is comprised of two parts: a SYNC period for synchronization signal, and a listen/sleep period. The listen/sleep period is again divided into multiple sub-slots. We call these wakeup slots. Each node is assigned a wakeup slot for data transmitting and receiving, and it can wake up only during its own wakeup slot. After its own wakeup slot, it goes to sleep until another wakeup slot is reached in the next superframe. The location of wakeup slot is assigned to each node depending on its ID (SID). For example, the location of wakeup slot can be determined by simply using the residual value of SID divided by the number of wakeup slots $\left(N_{w s}\right)$. The number of wakeup slots per listen/sleep period depends on applications used or network deployment. In high dense network, it may need a lot of wakeup slots. At this time, several nodes may share a single wakeup slot.

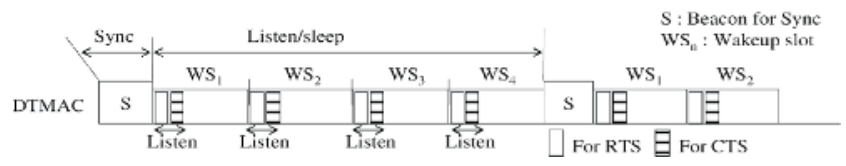

Fig. 1. Frame structure of our MAC protocol

All sensor nodes awake up during SYNC period and join in the contention to broadcast SYNC packet as done similarly in SMAC. The winner sends a SYNC packet to let its neighbor nodes know wakeup schedule information as well as to deal with clock drift [2]. On receiving a SYNC packet, each node knows the location of its own wakeup slots. Each sensor maintains a time synchronization and wakeup schedule information table for its neighboring nodes.

When a node has data to send, it looks up the wakeup schedule information table to find out the location of the wakeup slot assigned to the receiver, and waits until the wakeup slot comes. Upon seeing the receiver's wakeup slot, it starts RTS and CTS handshaking for transmission competition as performed similarly in SMAC. If it fails in the competition of RTS/CTS handshaking, it must wait for the next superframe.

As previously explained, in SMAC, every node joins the competition to transmit its data packet for every listen period. If a node fails in the competition, it must wait for the next listen time. Thus, it becomes the main cause of latency time problems. On the other hand, our MAC protocol can reduce energy consumption and minimize idle listening since it joins the competition only for its own short wakeup slot when the receiver is ready to receive its data.

\section{Simulation}

We evaluated the performance of our MAC protocol mechanisms through a computer simulation. The simulation parameters are listed in Table 1. To simplify the simulation, we assumed that the radio link propagation delay was zero without transmission 
error. Energy consumption model is based on real nodes: $0.016 \mathrm{~mW}$ while sleeping, $12.36 \mathrm{~mW}$ while idle listening, $12.50 \mathrm{~mW}$ while receiving, and $14.88 \mathrm{~mW}$ while transmitting a data packet [1].

The simulation was conducted in a static network with 9 sensor nodes as shown in Fig. 2. Each sending node, modeled as Constant Bit Rate (CBR) traffic source, had 20 packets. The number of wakeup slots was 4 . We assumed that the traffic flows to only one way from send nodes to the destination nodes through a unicast path. The simulation ran until every node sent all of its packets.

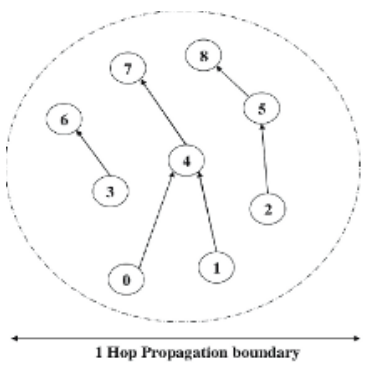

Fig. 2. Two-hop network topology for simulation
Table 1. Simulation parameters

\begin{tabular}{|l|l|}
\hline Radio bandwidth & $20 \mathrm{kbps}$ \\
\hline Contention window & 32 slot \\
\hline Data packet size & 150 Bytes \\
\hline RTS, CTS, ACK size & 20 Bytes \\
\hline Duration of beacon & $25 \mathrm{~ms}$ \\
\hline Frame interval & $625 \mathrm{~ms}$ \\
\hline SMAC listen duration( 10\% duty cycle) & $62.5 \mathrm{~ms}$ \\
\hline SWMAC wakeup slot duration & $150 \mathrm{~ms}$ \\
\hline SWMAC listen/sleep time duration & $25 \mathrm{~ms}$ \\
\hline
\end{tabular}

Fig. 3 shows the average number of collisions until each node sends all packets it has with different traffic load. For comparison, we implement a SMAC with adaptive listening, but we do not consider its synchronization and message passing scheme. In the SMAC with periodic sleep, each node is configured to operate on a $10 \%$ duty cycle. Also we implement a Carrier Sense Multiple Access/Collision Avoidance (CSMA/CA) MAC without periodical sleep schedule.

Our MAC protocol causes less collision than SMAC since each sensor node in our MAC protocol has a separate receiving time and it tries to send its packet only when the receiver is ready. In SMAC, on the other hand, if each node has the same duty cycle, they then join in contention of transmission at the same time. Thus, they can choose the same back-off time under heavy traffic load, which causes frequent collisions.

We compare the average packet queuing delay under various traffic loads for three MAC protocols, as illustrated in Fig. 4. In general, the queuing delay depends on the traffic load. In a heavy traffic case, queuing delay becomes a dominant factor in the latency of MAC protocol. In light traffic, there is no queuing delay since few packets are moving through the network. In the MAC protocol without sleeping, it immediately starts carrier sensing and tries to forward packets to the next hop. However, the MAC protocol with periodic sleeping, has an extra delay (called a 'sleep delay'), since when a sender gets a packet to transmit, it must wait until the receiver wakes up. Further, if the sender is defeated in a transmit competition it then must sleep until the next wakeup schedule time. This increases the queuing delay. However, our MAC protocol offers a lower queuing delay even under heavy traffic loads since it distributes competitions over the superframe. 
Fig. 5 shows the amount of energy consumed by all nodes in the network until the end of the simulation runs. We compared the total energy consumption of different MAC protocols under different traffic loads [2]. This figure shows that SMAC consumes more energy than our MAC protocol. This is because SMAC produces more retransmissions than our MAC protocol. However, both compared with the CSMA/CA can reduce total energy consumption using periodic listen/sleep schedule.

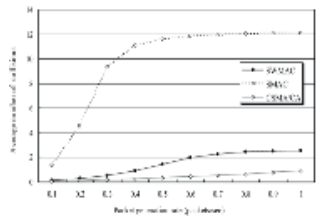

Fig. 3. Average number of collisions

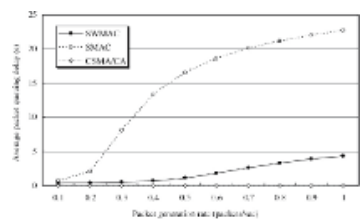

Fig. 4. Average packet queuing delay

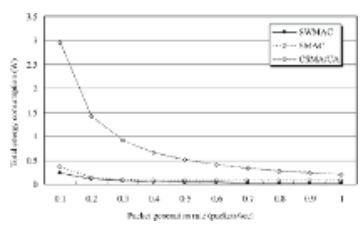

Fig. 5. Total energy consumption

\section{Conclusions}

We proposed a new MAC protocol, an energy efficient, low collision, and low latency MAC protocol using separate wakeup slots in the same duty cycle for wireless sensor networks. In our MAC protocol, each node joins the competition only for its own short wakeup slot when the receiver is ready to receive its data. Simulation results show that our MAC protocol can reduce probability of collisions and decrease sleep delay, which contributes to enhancing throughput and improving power efficiency.

Acknowledgements. This research is supported by Program for the Training of Graduate Students for Regional Innovation.

\section{References}

1. Curt Schurgers, Vlasios Tsiatsis, Saurabh Ganeriwal, Mani Srivastava : Optimizing Sensor Networks in the Energy-Latency-Density Design Space, IEEE Transactions on mobile computing, Vol. 1, No. 1, pp. 70-80, (2002)

2. W. Ye, J. Heidemann, and D. Estrin,: Medium Access Control with Coordinated, Adaptive Sleeping for ireless Sensor Networks, IEEE/ACM Transaction on Networking, Vol. 12, No.3, pp.493-506, (2004)

3. Gang Lu, Bhaskar Krishnamachari, Cauligi S. Raghavendra.: An Adaptive EnergyEfficient and Low-Latency MAC for Data Gathering in Wireless Sensor Networks, WMAN'04, Vol. 13, No. 13, pp. 224a, (2004)

4. Ramakrishnan, S. Huang, H. Balakrishnan, M. Mullen, J.: Impact of sleep in a wireless sensor MAC protocol, VTC2004-Fall, Vol. 7, pp. 4621-4624, (2004) 